\title{
Schichtdicken- und Temperaturabhängigkeit der Koerzitivkraft von dünnen Nickelaufdampfschidhten, gemessen mittels der magnetischen Widerstandsänderung
}

\author{
Von Wolfgang Hellenthal \\ Aus dem Physikalischen Institut der Universität Münster i. W. \\ (Z. Naturforschg. 14 a, 722-727 [1959]; eingegangen am 14. Mai 1959)
}

\begin{abstract}
Aus der Änderung des elektrischen Widerstandes im Magnetfeld wird die Koerzitivkraft von dünnen Nickelschichten, die auf Glasträgern von $20^{\circ} \mathrm{C}, 200^{\circ} \mathrm{C}$ und $400{ }^{\circ} \mathrm{C}$ aufgedampft wurden, gemessen. Bei den auf erhöhter Temperatur niedergeschlagenen Schichten ist die Koerzitivkraft im wesentlichen durch die Kristallanisotropie bedingt. Der Einfluß der Kristallitgröße (aus Elektronenbeugungsuntersuchungen) auf die gemessenen $H_{\mathrm{c}}$-Werte wird diskutiert. Die hohen Koerzitivkraftwerte von auf ungeheizter Unterlage niedergeschlagenen Schichten können durch das Auftreten von Spannungen erklärt werden.

Im Zusammenhang mit den Meßergebnissen der Schichtdicken- und Temperaturabhängigkeit von $H_{\mathrm{c}}$ bei den $200^{\circ} \mathrm{C}$-Schichten werden auch die möglichen Ursachen des Koerzitivkraftrückganges bei sehr dünnen Schichten behandelt. Es kommen hier sowohl die Verringerung der ferromagnetischen Anisotropiegrößen bei der Abnahme der spontanen Magnetisierung (infolge Schichtdickeneffektes) in Frage, als auch thermisch bedingte Schwankungserscheinungen.
\end{abstract}

Bei der Messung des elektrischen Widerstandes dünner ferromagnetischer Schichten in Abhängigkeit vom außen anliegenden Magnetfeld findet man den in Abb. 1 schematisch dargestellten Verlauf des Widerstandes. Der Kurvenverlauf bei kleinen Feldern ist durch die Orientierungsverteilung der Magnetisierungsvektoren in der Probe bestimmt. In diesem $\mathrm{Zu}$ sammenhang kann man den Minima des Longitudinaleffektes bzw. den Maxima des (in der Schichtebene) transversalen Effektes eine Koerzitivkraft $H_{\mathrm{c}}$ zuordnen. Auf diese Möglichkeit, aus Widerstandsmessungen die Koerzitivkraft dünner Schichten zu ermitteln, wurde bereits von IтTERвеEK und Mitarb. ${ }^{1}$ hingewiesen. Sie ist dann auch im Zusammenhang mit den Untersuchungen des Verfassers ${ }^{2,3}$ über die Schichtdicken- und Strukturabhängigkeit der magnetischen Widerstandsänderung an Nickel-Aufdampfschichten zur Ermittlung der magnetischen Größe $H_{\mathrm{c}}$ benutzt worden, deren Ergebnisse im folgenden mitgeteilt werden sollen. Ferner wurde die Methode auch von ReImer ${ }^{4}$ zur Untersuchung elektrolytisch niedergeschlagener Nickelschichten verwendet.

Bezeichnet man mit $\vartheta$ den Winkel zwischen Stromrichtung und Richtung des Magnetisierungsvektors in den einzelnen Domänen, so gilt für die pauschale Widerstandsänderung der Probe:

$$
\Delta R / R_{0}=A+B \overline{\cos ^{2} \vartheta} .
$$

1 A. van Itterbeek, R. Lambeir, B. Franken, G. J. van den Berg u. D. A. Loскноrst, Physica (Haag) 18, 137 [1952].

2 W. Hellenthal, Dissertation, Münster 1956.

3 W. Hellenthal, Z. Phys. 151, 421 [1958].
Die aus der Widerstandsänderung ermittelte Widerstandskoerzitivkraft ergibt sich im $R / H$-Diagramm (Abb. 1) an der Stelle, wo der quadratische Mittelwert $\overline{\cos ^{2} \vartheta}$ ein Minimum erreicht. Bei den üblichen magnetischen Methoden ergibt sich die Koerzitivkraft aus dem Verschwinden der pauschalen Magnetisierung $I$ der Probe, wobei gilt

$$
I=I_{\mathrm{s}} \overline{\cos \vartheta} .
$$

Wegen der unterschiedlichen Mittelwertsbildungen sind die beiden Koerzitivkraftdefinitionen verschieden und stimmen im allgemeinen nicht genau überein, was auch schon von ReImer ${ }^{4}$ diskutiert worden ist. Wenn im folgenden bei der Diskussion der Meßergebnisse die Koerzitivkraft dünnster Schichten behandelt wird, so ist hier stets die WiderstandsKoerzitivkraft gemeint.

Die Koerzitivkraft von dünnen Schichten (bzw. kleinen Teilchen) ist in einem Dickenbereich, wo die spontane Magnetisierung noch nicht merklich infolge Schichtdickeneffektes abgenommen hat (für Zimmertemperatur bei Nickel etwa $200 \AA$ ), gegenüber den Werten vom kompakten Material erhöht. Diese Erscheinung ist nach KitteL ${ }^{5,6}$ zu verstehen, wenn man annimmt, daß unterhalb einer kritischen Schichtdicke (bzw. Teilchengröße) Eindomänenkonfigurationen auftreten, bei denen die Ummagnetisie-

4 L. Reimer, Z. Naturforschg. 12 a, 558 [1957].

${ }^{6}$ C. Kittel, Rev. Mod. Phys. 21, 541 [1949].

5 C. Kittel, Phys. Rev. 70, 965 [1946]. 

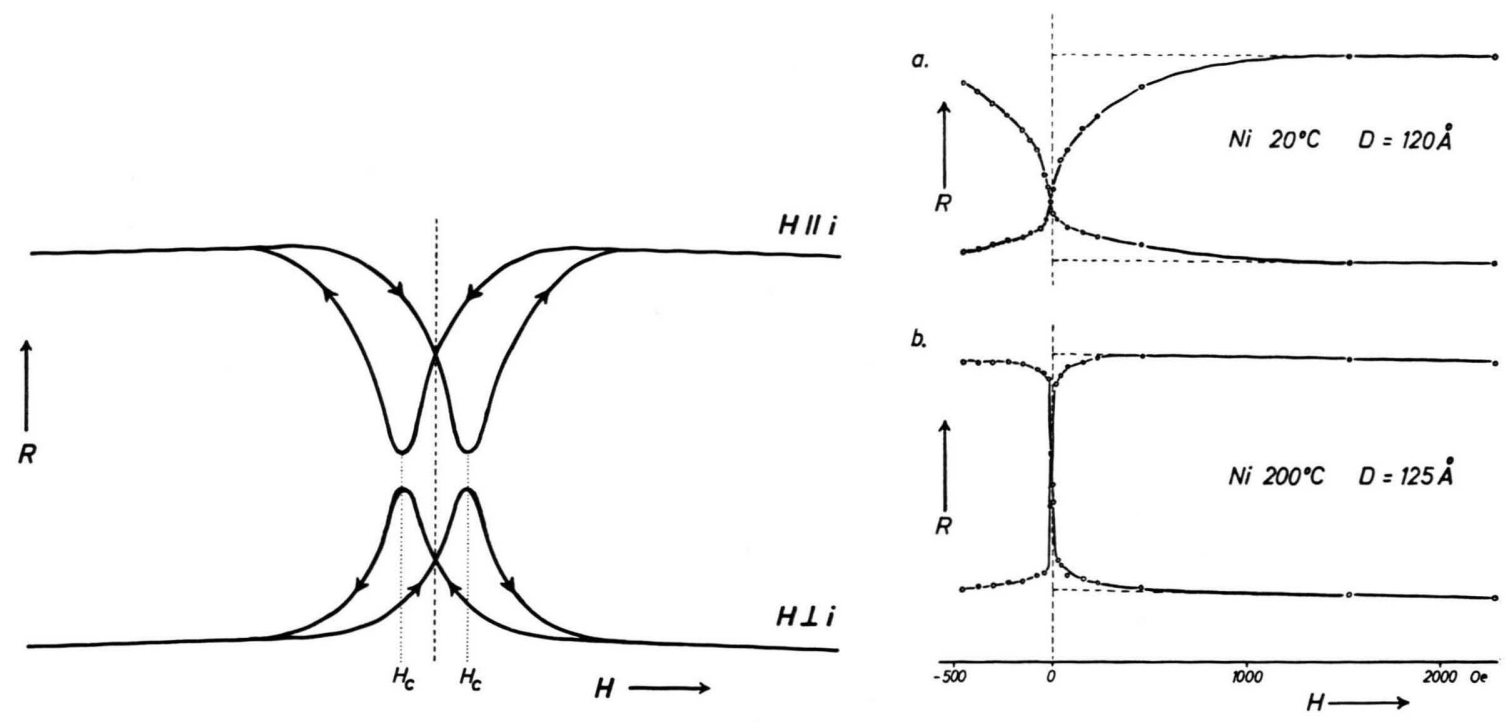

Abb. 1. Widerstand von Nickel in Abhängigkeit vom Magnetfeld, parallel und senkrecht zur Stromrichtung, beides in der Schichtebene (schematisch).

rung im wesentlichen nur durch Drehprozesse erfolgt, wofür höhere Gegenfelder erforderlich sind, als wenn Wandverschiebungen einen erheblichen Beitrag zur Ummagnetisierung liefern. Für das $\mathrm{Zu}$ standekommen der Koerzitivkraft sind in der Literatur drei Ursachen diskutiert worden:

a) die magnetokristalline Anisotropie,

b) Spannungen,

c) das Auftreten entmagnetisierender Felder (Gestaltsanisotropie).

Diese drei Fälle können sowohl einzeln als auch zusammen auftreten. Die Möglichkeit, experimentell festzustellen, welcher Anteil den wesentlichen Beitrag liefert, ergibt sich aus der Betrachtung der Formeln, welche für die drei Fälle gelten:

$$
H_{\mathrm{c}}=2 K / I_{\mathrm{s}}, \quad H_{\mathrm{c}}=3 \lambda \sigma / I_{\mathrm{s}}, \quad H_{\mathrm{c}}=\left(N_{\mathrm{t}}-N_{0}\right) I_{\mathrm{s}} .
$$

(Es bedeuten: $K=$ Kristallanisotropiekonstante, $\lambda=$ Sättigungsmagnetostriktion, $\sigma=$ Spannungen, $N_{\mathrm{t}}$ und $N_{0}$ sind die Entmagnetisierungsfaktoren transversal und longitudinal, $I_{\mathrm{S}}=$ spontane Magnetisierung.)

Diese Beziehungen sind abgeleitet unter der Voraussetzung, daß es sich um einzelne, magnetisch isolierte Teilchen handelt, deren Vorzugsrichtung dem von außen angelegten Gegenfeld gerade entgegengesetzt gerichtet ist. Da bei den vorliegenden Schichten die Vorzugsrichtungen nicht einachsig sind und außerdem keine isolierten Bereiche vorliegen, ist eine quantitative Gültigkeit der Gln. $(3 a-c)$

Abb. 2. Vergleich von Widerstandskurven an Nickelschichten; oben: große Spannungen, unten: keine wesentlichen Spannungen.

nicht zu erwarten. Sie können jedoch qualitativ benutzt werden, um die verschiedenen Koerzitivkraftursachen zu trennen, und zwar mit Hilfe der Temperaturabhängigkeit. Dies gilt vor allem für a) und c) ${ }^{7}$. Der Fall b) ist zwar auch mittels der Temperaturabhängigkeit von $H_{\mathrm{c}}$ nach a) und c) zu trennen, leichter jedoch meistens schon an der Form der Magnetisierungskurve (bzw. Widerstandskurve, vgl. Abb. 2) zu erkennen.

Sieht man einmal von den schon genannten strukturbedingten Schwierigkeiten der Anwendung der Gln. $(3 \mathrm{a}-\mathrm{c}) \mathrm{ab}$, so sind bei sehr dünnen Schichten (um $100 \AA$ und darunter) und bei sehr kleinen Teilchen diese Gleichungen grundsätzlich auch zur Beschreibung der drei Koerzitivkraftursachen geeignet, jedoch muß man hierbei berücksichtigen, daß:

$1 \alpha$ ) die spontane Magnetisierung gegenüber den Werten des kompakten Materials erniedrigt ist (Lit. hierzu bei MAYER ${ }^{8}$ ) und

$1 \beta$ ) im Zusammenhang mit dem durch geringe Schichtdicke bedingten Rückgang der spontanen Magnetisierung auch eine Abnahme der ferromagnetischen Anisotropiegrößen (Kristallenergie, Sättigungsmagnetostriktion, Widerstandsanisotropie) zu erwarten ist ${ }^{9}$, wobei diese Größen bei der jeweiligen (von der Schichtdicke abhängigen) Curie-Tempera-

7 L. WeIl u. S. Marfoure, J. Phys. Radium 8, 358 [1947].

8 H. MAYER, Physik dünner Schichten, Bd. II, Wissenschaftliche Verlagsgesellschaft, Stuttgart 1955.

9 W. Hellenthal, Phys. Verh. 7, 163 [1956]. 
tur ${ }^{10-12}$ verschwinden müssen. Aus diesem Grunde ist für die genannten Größen auch eine andere Temperaturabhängigkeit als beim kompakten Material zu erwarten.

2) Neben den vorgenannten zeitunabhängigen Effekten kann bei sehr dünnen Schichten auch mit dem Auftreten thermisch bedingter Richtungschwankungen der Magnetisierungsvektoren der einzelnen Bereiche gerechnet werden. Eine quantitative Anwendung der NÉELschen Theorie ${ }^{13}$, die für magnetisch isolierte Teilchen gilt, ist im Falle zusammenhängender Schichten nicht möglich.

\section{Experimentelles}

Die für die vorliegende Untersuchung verwendeten Nickelschichten wurden auf Glasträger bei verschiedenen Temperaturen $\left(20{ }^{\circ} \mathrm{C}, 200{ }^{\circ} \mathrm{C}\right.$ und $\left.400{ }^{\circ} \mathrm{C}\right)$ in gleicher Weise aufgedampft und anschließend sofort nach Herstellung mit einer SiO-Deckschicht zum Schutz gegen Oxydation versehen, wie vom Verfasser bereits an anderer Stelle ${ }^{3}$ beschrieben.

Die Anordnung zur Messung des elektrischen Widerstandes im Magnetfeld bestand aus einer Wheatstoneschen Brücke. Die Aufnahme der Widerstandskurven erfolgte nach der Ausschlagsmethode. Das Magnetfeld wurde mit einer wassergekühlten Zylinderspule erzeugt, in die noch ein Ofen bzw. zur Messung bei tiefen Temperaturen ein Dewargefäß eingesetzt werden konnte (Schema der Anordnung in ${ }^{14}$ ).

Zur Kristallitgrößenbestimmung sind Elektronenbeugungsaufnahmen von Schichten möglichst ähnlicher Struktur gemacht worden, die auf SiO-Häutchen in gleicher Weise wie die Schichten auf den Glasträgern aufgedampft und wieder mit $\mathrm{SiO}$ überdeckt waren. Die Bestimmung der mittleren Kristallitgröße erfolgte jeweils aus der photometrisch ermittelten Linienbreite der Debye-Scherrer-Aufnahmen, wobei die Annahme gemacht ist, daß als wesentliche Verbreiterungsursache die Teilchenkleinheit wirksam ist.

\section{Meßergebnisse und Diskussion}

Die Ergebnisse der Koerzitivkraftmessungen der bei den drei verschiedenen Trägertemperaturen hergestellten Nickelaufdampfschichten sind in Abb. 3 dargestellt. Allen Kurven gemeinsam ist ein starker Abfall der Werte unterhalb $100-200 \AA$. Da in diesem Bereich mit den unter $1 \alpha, \beta$ ) und 2) diskutierten Einflüssen zu rechnen ist, müssen für die Frage

10 A. Drigo, Nuovo Cim. 8, 498 [1951].

11 E. C. Crittenden jr. u. R. W. Hoffman, Rev. Mod. Phys. 25, 310 [1953].

12 W. Hellenthal, Z. Naturforschg. 13 a, 566 [1958]. nach der Koerzitivkraftursache die dickeren Schichten (Schichtdicke > $200 \AA$ ) herangezogen werden.

Die großen Koerzitivkraftwerte der $20{ }^{\circ} \mathrm{C}$-Schichten können durch das Auftreten erheblicher Spannungen in diesen Proben erklärt werden. Die Annahme solcher Spannungen wird auch durch die Kurvenform (Abb.2) der Widerstandskurve sowie

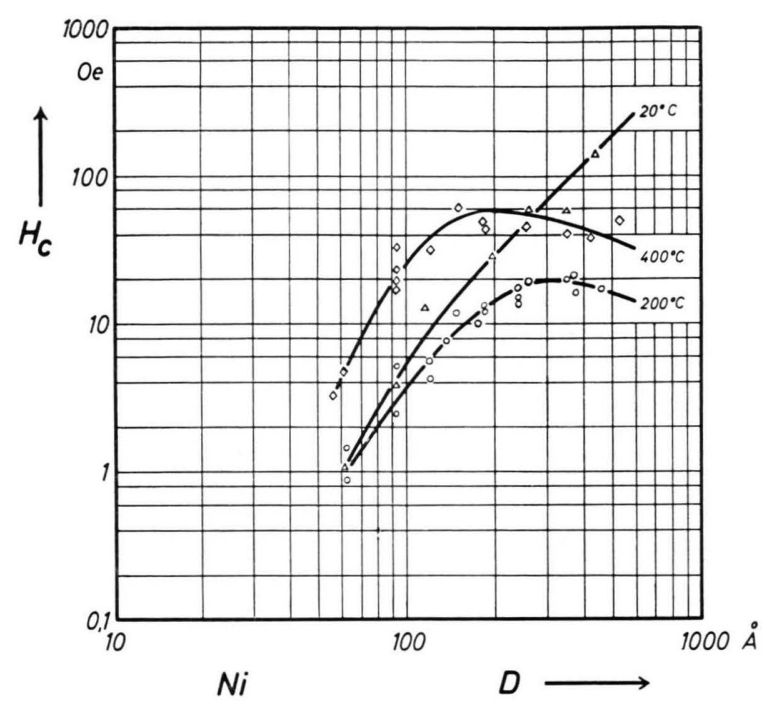

Abb. 3. Schichtdickenabhängigkeit der aus dem Widerstands. effekt ermittelten Koerzitivkraft an Proben verschiedener Herstellungsbedingungen.

durch die Orientierungsverteilung der Magnetisierungsvektoren ${ }^{14}$ in derartigen Schichten nahegelegt. Auch die von Reimer ${ }^{15}$ ballistisch ermittelten anderen magnetischen Größen an Schichten ähnlicher Herstellungsbedingungen (auf feinkristallinem Kupfer aufgedampft) führen zu der gleichen Auffassung.

Bei den auf geheizten Trägern niedergeschlagenen Schichten ist die Koerzitivkraft durch die Kristallenergie bedingt, denn die beobachtete Vergrößerung der $H_{\mathrm{c}}$-Werte um einen Faktor 4 bis 5 (vgl. auch Abb. 5) bei einer Senkung der Meßtemperatur von $20{ }^{\circ} \mathrm{C}$ auf $-195{ }^{\circ} \mathrm{C}$ läßt sich nur nach Gl. (3 a) verstehen auf Grund der starken Temperaturabhängigkeit von $K$. Bei dieser Temperaturerniedrigung nimmt die Kristallenergie $K$ um etwa einen Faktor 5 zu, während die spontane Magnetisierung nur um einen Faktor 1,1 größer wird (vgl. auch ReImeR ${ }^{16}$ ). Bezüglich der Maximalwerte der $H_{\mathrm{c}}$-Kurven der

${ }^{13}$ L. NÉEL, Ann. Géophys. 5, 99 [1949].

14 W. Hellenthal, Z. Phys. 153, 359 [1958].

15 L. Reimer, Z. Naturforschg. 12 a, 550 [1957].

16 L. Reimer, Z. Naturforschg. 11 a, 649 [1956]. 
$200{ }^{\circ} \mathrm{C}$ - und $400{ }^{\circ} \mathrm{C}$-Schichten in Abb. 3 ist ein Vergleich mit den Ergebnissen von ReIMER ${ }^{15,16}$ an elektrolytisch auf Cu-Aufdampfschichten hergestellten Proben möglich. Die Größe des dort gefundenen Maximums liegt fast so hoch wie bei den $400^{\circ} \mathrm{C}$ Schichten des Verfassers. Die dort elektronenmikroskopisch bestimmte mittlere Kristallitgröße der Proben liegt auch in der Größenordnung der vom Verfasser mittels Elektronenbeugung ermittelten Werte (vgl. Abb. 4). Die sehr viel höheren KoerzitivkraftWerte, die Reimer bei seinen elektrolytisch auf Elektrolytkupfer abgeschiedenen Nickelschichten fand $\Lambda$ $(200-250 \mathrm{Oe})$, sind durch die dort aufgetretene Lamellenstruktur zu erklären, bei der die Koerzitivkraft durch Formanisotropie bedingt ist. Dieser Fall trat jedoch bei den vom Verfasser untersuchten Schichten nicht auf, was aus der Temperaturabhängigkeit der $H_{\mathrm{c}}$-Werte geschlossen wurde.

Der Unterschied der Werte zwischen den $200{ }^{\circ} \mathrm{C}$ und den $400^{\circ} \mathrm{C}$-Schichten kann an Hand der durch Elektronenbeugung bestimmten mittleren Kristallitgröße $\Lambda$ (Abb. 4) als Struktureinfluß gedeutet werden. Die geringere Kristallitgröße der $200^{\circ} \mathrm{C}$-Schichten läßt eine relativ stärkere magnetische Kopplung benachbarter Bereiche erwarten und damit auch leichtere Drehbarkeit bzw. geringere Koerzitivkraft bei sonst gleicher Koerzitivkraftursache.

Betrachtet man nun den Dickenbereich $(D<200 \AA)$, in dem der starke Rückgang der Koerzitivkraftwerte beobachtet wird, so zeigen die Elektronenbeugungsaufnahmen, daß die Kristallitgröße bei jeweils festen Herstellungsbedingungen nur wenig variiert. Demnach müssen andere Ursachen, z. B. die unter $1 \beta$ ) und 2) genannten, berücksichtigt werden. Eine Trennung der Effekte nach $1 \beta$ ) und 2) wäre einerseits über die Zeitabhängigkeit möglich, andererseits in beschränktem Umfange auch über die Temperaturabhängigkeit, denn der exponentielle Zusammenhang zwischen Relaxationszeit und Temperatur ${ }^{13}$ würde bei vorgegebener Meßzeit nach Überschreiten einer kritischen Temperatur (bei Teilchen einheitlicher Größe) einen außerordentlich starken Rückgang der Koerzitivkraft erwarten lassen. Um hier weitere Aufschlüsse zu erhalten, wurde nunmehr die Koerzitivkraft von $200^{\circ} \mathrm{C}$-Schichten bei verschiedenen Meßtemperaturen ermittelt (Abb. 5). Aus den Messungen geht hervor, daß auch bei den dünnsten Schichten Koerzitivkräfte in der Größenordnung von 100 Oe auftreten, sofern die Meßtemperatur niedrig genug ist. Der mit steigender Temperatur einsetzende
$H_{\mathrm{c}}$-Abfall konnte über etwa zwei Zehnerpotenzen verfolgt werden. Der Koerzitivkraft-Temperatur-Verlauf der dicksten Schicht $(300 \AA)$ ist etwa so, wie man ihn entsprechend der Temperaturabhängigkeit

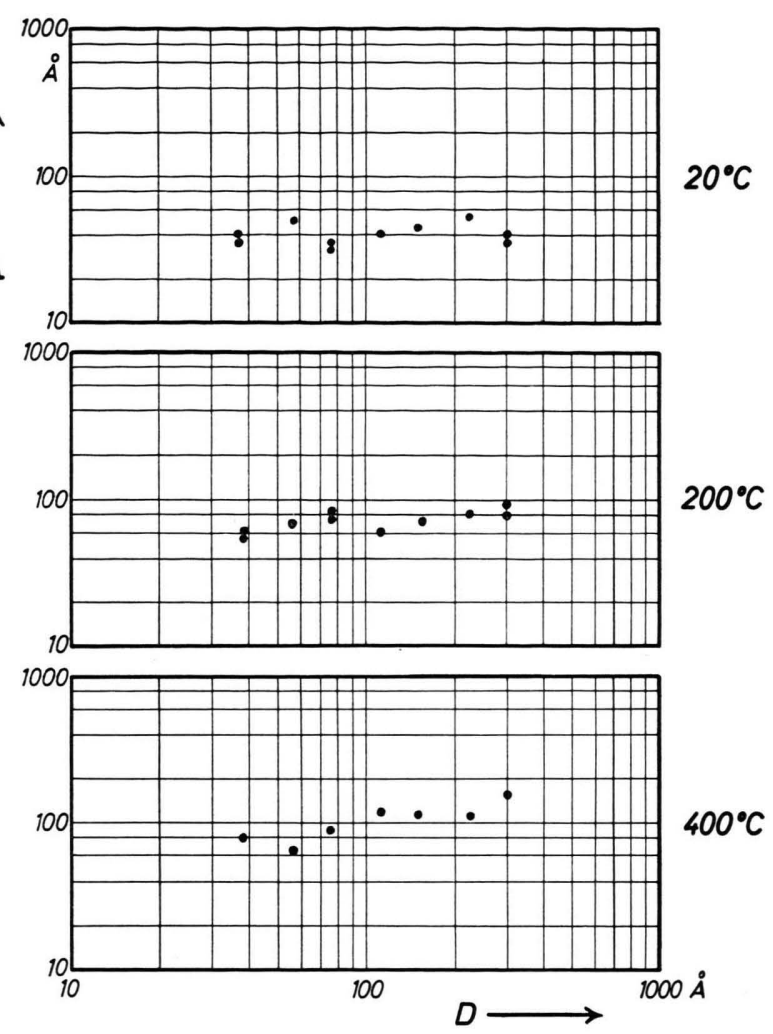

Abb. 4. Mittlere Kristallitgröße $\Lambda$ von Nickelaufdampfschichten verschiedener Herstellungsbedingungen, ermittelt aus Elektroneninterferenzen.

der Kristallanisotropiekonstanten des kompakten Materials erwarten kann. Bei dünneren Schichten bzw. erhöhten Temperaturen wurden im Verlauf der Messungen nur geringe Zieherscheinungen beobachtet. Daraus kann man schließen, daß zwar die unter 2) diskutierten Zeiteffekte (Schwankungserscheinungen) einen Einfluß auf die Koerzitivkraft haben, jedoch zunächst die unter $1 \beta$ ) erwähnten Effekte hierfür heranzuziehen sind. Das steht auch im Einklang mit den Ergebnissen einer Untersuchung des Verfassers über die Frequenzabhängigkeit der Koerzitivkraft bei solchen Schichten ${ }^{17}$.

$\mathrm{Da}$ es sich bei den untersuchten Schichten von $200{ }^{\circ} \mathrm{C}$ Herstellungstemperatur um die durch die Kristallanisotropie bedingte Koerzitivkraft handelt,

17 W. Hellenthal, Phys. Verh. 9, 214 [1958]. 

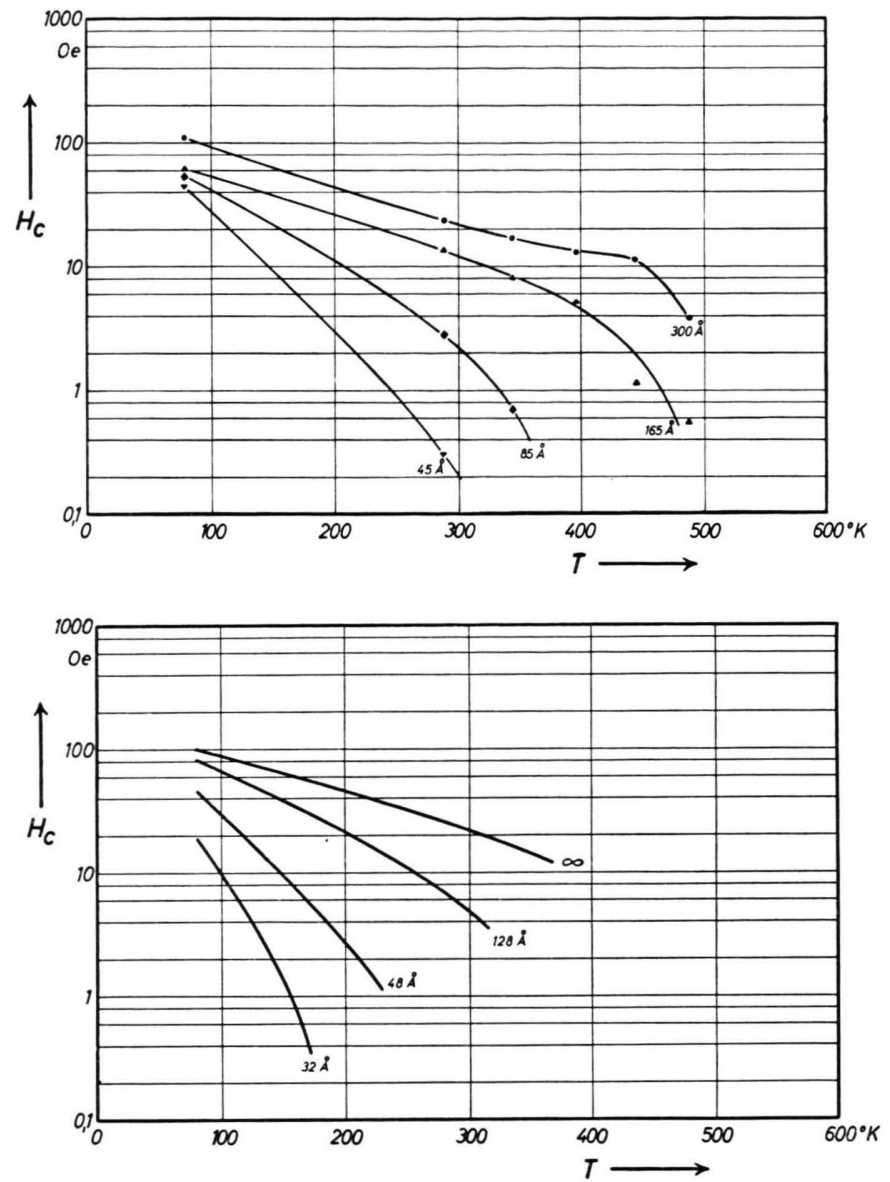

Abb. 5. Temperaturabhängigkeit der Koerzitivkraft von Nickelschichten verschiedener Dicke $\left(200^{\circ} \mathrm{C}\right.$ Trägertemperatur beim Aufdampfen).
Abb. 6. Für dünne Nickelschichten berechnete Temperatur- und Dickenabhängigkeit der Koerzitivkraft. erschien es interessant, in Analogie zu dem bei den theoretischen Arbeiten (z. B. CARR ${ }^{18}$, dort auch weitere Literatur) behandelten Zusammenhang zwischen der (temperaturabhängigen) spontanen Magnetisierung des kompakten Materials und der Kristallanisotropie auch einen entsprechenden Ansatz für die durch Schichtdickeneffekt erniedrigte spontane Magnetisierung durchzuführen. Man kann z. B. annehmen, daß entsprechend der Formel von CARR ${ }^{18}$ die Kristallanisotropie allgemein durch die spontane Magnetisierung bestimmt ist, unabhängig davon, ob letztere durch Temperatur- oder Schichtdickeneffekt erniedrigt ist. Die genannte Formel lautet:

$$
\frac{K(T)}{K(0)}=\left(1-1,74 \frac{T}{T_{\mathrm{c}}}\right)\left(\frac{I_{\mathrm{s}}}{I_{0}}\right)^{10}
$$

Für die Curie-Temperatur $T_{\mathrm{c}}$ wurde der zur jeweiligen Schichtdicke gehörige Wert eingesetzt, wie er

18 W. J. Carr Jr., Phys. Rev. 109, 1971 [1958].

19 M. Klein u. R. Smith, Phys. Rev. 81, 378 [1951]. nach einem vom Verfasser angegebenen Verfahren ${ }^{12}$ an solchen Schichten ermittelt wurde. Die spontane Magnetisierung $I_{\mathrm{s}}$ wurde nach der von DRIGO ${ }^{10}$ und Crittenden $^{11}$ empirisch modifizierten Theorie von KLein und SMITH ${ }^{19}$ berechnet und eingesetzt. Abb. 6 enthält die aus Gl. ( $3 \mathrm{a})$ berechneten $H_{\mathrm{c}}$-Werte mit den nach Gl. (4) berechneten $K$-Werten. Dabei sind die Kurven der Abb. 6 auf den für eine $300 \AA$ Schicht gemessenen Verlauf bezogen und der bei dieser Schicht gefundene Verlauf gleich dem des kompakten Materials gesetzt worden.

Der Vergleich der in Abb. 6 dargestellten berechneten Werte mit Abb. 5 zeigt, daß die Carrsche Formel die beobachteten Werte größenordnungsmäßig richtig wiedergibt. Die berechneten Werte zeigen eine etwas stärkere Temperaturabhängigkeit als die gemessenen. Die Meßwerte sind auch noch durch die in der vorliegenden Untersuchung nicht abgetrennten Zeiteffekte erniedrigt. Daher wäre, wenn man nur die nach $1 \beta$ ) verringerten $H_{\mathrm{c}}$-Werte (größer als 
die gemessenen) zum Vergleich zur Verfügung hätte, der Unterschied noch etwas größer als er aus dem Vergleich von Abb. 5 und Abb. 6 folgt. Außerdem kann man aus der Tatsache, daß die dünneren Schichten etwas kleinere Korngröße haben (vgl. Abb. 4), einen weiteren kleinen $H_{\mathrm{c}}$-erniedrigenden Beitrag erwarten (vgl. Abb. 3). Zusammenfassend kann man also sagen, daß der auf die beschriebene Art berech- nete $H_{\mathrm{c}}$-Abfall infolge schichtdickenbedingter Verringerung der Kristallanisotropie etwas steiler verläuft, als nach den Messungen dem Einfluß eines solchen Effektes zugeschrieben werden kann.

Herrn Professor Dr. E. KAPPLER möchte ich für das dieser Arbeit entgegengebrachte Interesse und die Bereitstellung von Institutsmitteln danken.

\title{
Über den Leitungsmechanismus oxydischer Halbleiter bei hohen Temperaturen
}

\author{
Von J. Rudolph \\ Aus der Osram-Studiengesellschaft Augsburg \\ (Z. Naturforschg. 14 a, 727-737 [1959]; eingegangen am 2. Juni 1959)
}

\begin{abstract}
Die Leitfähigkeit $\sigma$ oxydischer Halbleiter in Gegenwart von Sauerstoff wird bei hohen Temperaturen und nicht $\mathrm{zu}$ großen Störstellenkonzentrationen nicht allein durch die thermische Abspaltung von Trägern aus den Störstellen, sondern in entscheidendem Maße auch durch eine von der Temperatur $T$ und vom Sauerstoffdruck $P_{\mathrm{O}_{2}}$ abhängige Veränderung der Störstellenkonzentration als Folge einer Wechselwirkung mit der umgebenden Atmosphäre bestimmt.

Bei Oxyden von Kationen mit abgesättigter Valenz sind dabei zwei Verhaltensweisen kennzeichnend: (a) Zunehmende Aufnahme von überschüssigem Sauerstoff im Kristall mit steigendem Druck - trotz begrenzter Kationenvalenz - und Ausbildung einer p-Leitung $\left(\sigma \sim P_{0_{2}}{ }^{1 / x}\right)$; (b) Dissoziation unter Abspaltung von Sauerstoff und Ausbildung einer n-Leitung $\left(\sigma \sim \mathrm{P}_{\mathrm{O}_{2}}-1 / x\right)$. Wie an Hand von Leitfähigkeitsmessungen als Funktion der Temperatur und des Sauerstoffdruckes sowie auf Grund von Thermokraftmessungen gezeigt wird, sind typische Vertreter für (a): $\mathrm{BaO}, \mathrm{SrO}, \mathrm{CaO}$, $\mathrm{ThO}_{2}, \mathrm{ZrO}_{2}$ und $\mathrm{La}_{2} \mathrm{O}_{3}$, für (b) z. B. $\mathrm{CeO}_{2}, \mathrm{TiO}_{2}$ und $\mathrm{ZnO}$. Durch Dotierung mit anderswertigen Kationen können unter normalen Meßbedingungen p-leitende Oxyde der Gruppe (a) auch in n-leitendem Zustand sowie normalerweise n-leitende Oxyde der Gruppe (b), z. B. $\mathrm{TiO}_{2}$ und $\mathrm{ZnO}$, auch im p-leitenden Zustand erhalten werden. In eng durch $T$ und $P_{\mathrm{O}_{2}}$ begrenzten Bereichen treten dabei auch Eigenleitungszustände auf.

Es wird ein allgemeines, aus dem Massenwirkungsgesetz abgeleitetes Schema von LeitfähigkeitsSauerstoffdruck-Isothermen angegeben, das viele Einzelheiten im Erscheinungsbild der oxydischen Halbleiter zu erklären vermag.
\end{abstract}

Entsprechend der Klassifizierung der oxydischen Halbleiter ${ }^{1,2,3}$ sind auf Grund ihres Verhaltens drei Halbleiterhauptgruppen zu unterscheiden: 1. Reduktionshalbleiter, wie z. B. $\mathrm{ZnO}, \mathrm{CdO}, \mathrm{TiO}_{2}$ u. a., bei denen infolge eines im allgemeinen leicht zu erreichenden Sauerstoffausbaus aus dem Kristall eine Elektronenüberschußleitung beobachtet wird; 2. Oxydationshalbleiter, wie z. B. $\mathrm{Cu}_{2} \mathrm{O}, \mathrm{NiO}, \mathrm{UO}_{2}$, die infolge der Neigung der Metallionen zur Bildung höherwertiger Ionen überschüssigen Sauerstoff aufzunehmen vermögen und damit eine Elektronenmangelleitung zeigen; 3. Superoxydationshalbleiter, wie z. $\mathrm{B} . \mathrm{BaO}$ und $\mathrm{CaO}$, in die trotz der begrenzten Valenz der Erdalkalien Sauerstoff über die Stöchiometrie hinaus eingebaut werden kann und die daher

1 W. Sсноттку, Z. Elektrochem. 45, 33 [1939].

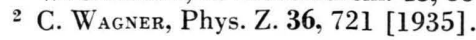

ebenfalls in Gegenwart von Sauerstoff eine erhöhte Leitfähigkeit mit den charakteristischen Merkmalen einer p-Leitung aufweisen.

In Erweiterung früherer Untersuchungen ${ }^{4}$ über das typische Verhalten der Vertreter dieser 3. Gruppe, $\mathrm{BaO}$ und $(\mathrm{Ba}, \mathrm{Sr}) \mathrm{O}$, hat sich nun gezeigt, daß das die Superoxydationshalbleiter kennzeichnende Verhalten nicht auf die Erdalkalioxyde beschränkt ist. Vielmehr vermögen auch eine Reihe anderer Oxyde, bei denen jedoch eine ausgesprochene Neigung zur Bildung von Peroxydverbindungen nicht bekannt ist, trotz begrenzter Kationenvalenz überschüssigen Sauerstoff aufzunehmen, wodurch dann ein Halbleiterverhalten ganz entsprechend dem der Erdalkalioxyde verursacht wird.

3 W. Meyer, Z. Phys. 85, 278 [1933].

4 J. Rudolph, Z. Naturforschg. 33 a, 757 [1958]. 